\title{
IMPLANTATION OF MICRO-CIRCUITS FOR MYO-ELECTRIC CONTROL OF PROSTHESES
}

\author{
P. Herberts, R. Kadefors, Göteborg, Sweden, E. Kaiser, Copenhagen, Denmark, \\ and I. Petersén, Göteborg, Sweden
}

From the Departments of Clinical Neurophysiology* and Orthopaedic Surgery, $\dagger$ Sahlgrenska sjukhuset, the Research Laboratory of Medical Electronics, $\ddagger$ Chalmers University of Technology, Göteborg, Sweden, and the Kaiser Laboratory, Copenhagen, Denmark

The possibility of using myo-electric potentials for the control of a powered prosthesis was first demonstrated by Battye, Nightingale and Whillis (1955). A working myo-electric control system was demonstrated, under laboratory conditions, by Kobrinski in Moscow (Kobrinski et al. 1961). At present three myo-electrically controlled hand prostheses are commercially available: Viennatone in Austria, INAIL in Italy, and a Russian unit.

Myo-potentials can be detected by means of surface electrodes and needle electrodes. The needle electrodes, routinely used in clinical practice, cannot be used in the control of prostheses because of pain and the risk of infection. Myo-electric hand prostheses have, until now, been operated by surface electrodes. Many problems associated with surface electrode techniques have recently been studied by Kadefors, Herberts, Hirsch, Kaiser and Petersén (1968). In long-term use surface electrodes often cause skin irritation, which is more pronounced with wet than with dry electrodes. Electrode movements and displacements cause errors in potentials. The ratio of signal to noise and the amount of high frequency power in the signals detected with present surface electrode techniques are inadequate for the proportional control of a myo-electric system.

Myo-potentials can also be recorded by a thin wire inserted into the muscle percutaneously by means of a cannula. This technique has been used in animal experiments for many years, and more recently in man by Scott (1965) and Caldwell (1967). These types of electrodes were discussed by Basmajian (1967).

A recent technique, employing telemetry, has involved the implantation of a miniature electronic transponder under the skin in close contact with the muscle. Appropriate microcircuits have been developed by Ko (1964) and independently by Hirsch, Kaiser and Petersén (1966), who published the first results from implantation of micro-circuits in man.

The requirements of an implantable electrode are: 1) that it produces minimal foreignbody reaction and is non-toxic and non-carcinogenic; 2) that it is capable of sterilisation; 3 ) that it is easy to implant and painless in use; 4) that it is reliable and capable of providing reproducible signals; 5) that it is miniaturised as much as possible; 6) that it is chemically inert and withstands the body environment for a long time; and 7) that it is maintenance-free.

In 1964 Hirsch, Kaiser and Petersén started research in the field of myo-electric control of prostheses. As a part of this programme an implantable electrode was developed. On the basis of their work a refined type of electrode has now been manufactured (Hirsch, Kadefors, Kaiser and Petersén 1967) and will be discussed in this paper. Several of these electrodes have been implanted in volunteer normal subjects and below-elbow amputees. The signals have been analysed immediately after implantation and subsequently at regular intervals.

\section{TECHNICAL DESCRIPTION}

External energising of the electrode by electromagnetic induction has been chosen as the most suitable method. The system therefore consists of two parts: one external to the body for energy

$$
\text { * Head, Ingemar Petersén. † Head, Carl Hirsch. } \ddagger \text { Head, Henry Wallman. }
$$


transmission and information detection, and the other implanted for energy conversion and transmission of information in modulated form (Fig. 1).

The energy source is a $3 \mathrm{MHz}$ oscillator with an output power of approximately $30 \mathrm{~mW}$., providing an almost uniform electromagnetic field in the environment of the implanted electrode, a circuit diagram of which is shown in Figure 2. In the energy receiver coil of this circuit (to the right)

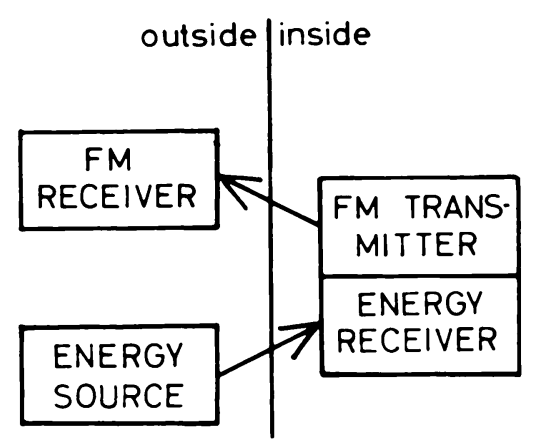

Fig. 1

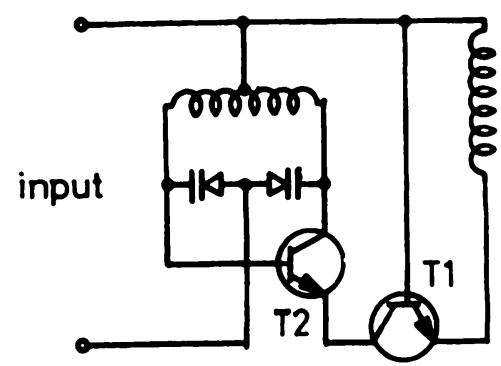

FIG. 2

Figure 1-A diagram showing the principle of the telemetry system. The implanted transmitter (on the right) is electromagnetically coupled to an external high frequency energy source (on the left). The myo-potentials modulate the frequency of oscillation of the implanted transmitter (Hirsch, Kaiser and Petersén 1964). Figure 2-A diagram of the circuit of the implant electrode.

an alternating current is induced. It is necessary to convert this signal to a direct current which is sufficient to bias the active elements of the transmitter part of the implant. Such a conversion is achieved by extensive use of the internal parameters of transistor T1 (Kadefors, Kaiser and Petersén 1968). Over the base-collector junction a direct current voltage of approximately $0.5 \mathrm{~V}$. is developed, smoothed and also stabilised. The transmission part of the implant is a Hartley oscillator with transistor $\mathrm{T} 2$ as the active element and a centre frequency of approximately $450 \mathrm{kHz}$. The resonance circuit consists of a centre-tapped inductance coil and two voltage-dependent capacitors in a bridge configuration. When myo-electric potentials are applied to the input terminals (to the extreme left in Figure 2) the capacitance values are changed and the oscillator's frequency is thus modified. The inductance coil of the Hartley circuit acts as a transmitter. This signal is detected and processed in the external circuit.

Since semiconductors are extremely sensitive to the ingress of impurities, the reliability of the implanted circuit is dependent on the rigorous exclusion of body fluids. Therefore, semiconductors have been bonded in " flat-pack " capsules of the type used for the hermetic encapsulation of monolithic integrated circuits. These capsules are made of gold-plated covar and glass. They measure $6 \times 3 \times 1 \mathrm{~mm}$. and are sealed by soldering with tin.

The coils of the implanted circuits are wound perpendicularly on a ferrite core. They are of high electrical reliability but require mechanical protection.

Connection with the body tissues has been obtained by means of gold wires, $0.5 \mathrm{~mm}$. in diameter and $6 \mathrm{~mm}$. in length which are soldered to the "flat-pack" connectors. Thus the principal materials of the implant are: gold, glass, covar, tin, copper and ferrite. As the body should be protected from direct contact with at least the active metals, the implants are encapsulated in CIBA Araldite D (CY 230) catalysed by hardener HY 956. Araldite $D$ belongs to the epoxy resin family; it is based upon epichlorhydrine and bisfenol-A and contains also a plasticiser for the prevention of cracking after curing. Hardener HY 956 is a modified aliphatic polyamine having low viscosity and promoting curing at room temperature. The resulting plastic is almost crystal clear, is of high mechanical, electrical and chemical stability and has a very low toxicity. It can withstand temperature shocks of at least 150 degrees Centigrade, and thus can be sterilised by heat. Water vapour diffusion is, however,

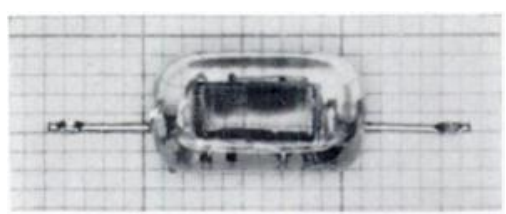

FIG. 3

The electrode prepared for implantation. The gold wires serve as tissue terminals. In the encapsulated part of the transmitter the coils can be seen, wound perpendicularly on a ferrite core. considerable and thus the resin cannot provide total protection for the embedded semiconductors.

An encapsulated electrode is shown in Figure 3. The Araldite layer is $0.5-1 \mathrm{~mm}$. in thickness, resulting in an overall size of approximately $5 \times 11 \times 4 \mathrm{~mm}$. The electrode satisfies the recommended 
requirement that it should be sterilisable by heat and readily survives the 30 minute cycles to 120 degrees Centigrade in dry air. Approximate electrical characteristics are: input impedance $2 \mathrm{nF}$ in parallel with $10 \mathrm{Mohm}$; bandwidth with the present detector $1 \mathrm{kHz}$; noise level $1 \mu \mathrm{Vr} . \mathrm{m} . \mathrm{s}$. referred to input and measured at room temperature with a bandwidth of $400 \mathrm{~Hz}$. The maximum working distance between energy source and implanted transmitter is about $15 \mathrm{~mm}$., which is adequate in the case of subcutaneous implantation.

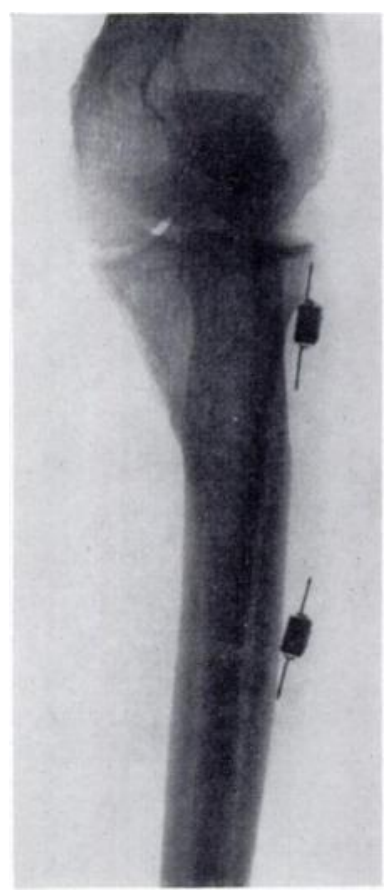

Fig. 4

Two electrodes implanted subcutaneously on the extensor side of a normal forearm (Case 1).

\section{CLINICAL MATERIAL AND METHOD}

The electrodes have been implanted in two healthy volunteers and in two below-elbow amputees, also volunteers.

Case 1-A thirty-two-year-old male technical assistant in the operation theatre of the orthopaedic clinic. In November 1966 two electrodes were implanted subcutaneously over the muscles on the extensor side of the left forearm. The electrodes were placed immediately superficial to the fascia of the extensor carpi radialis longus muscle with the gold wires along the axis of the muscle fibres and with five centimetres distance between the electrodes (Fig. 4).

At first the function of the electrodes was tested every month and then at regular intervals of two months. Five months after the implantation the more distally placed electrode was removed for technical control and histological examination of the tissue reactions that had occurred. After fifteen months the remaining electrode was removed.

Case 2-A twenty-eight-year-old female nurse. In January 1967 two electrodes were implanted subcutaneously on the extensor and flexor sides of the right forearm. One electrode was placed superficial to the fascia of the extensor carpi radialis longus muscle and the other superficial to the fascia of the flexor digitorum communis muscle. After nine months the electrode placed on the flexor side of the forearm was removed, the other one thirteen months after the implantation.

Case 3-The first below-elbow amputee was a twenty-six-year-old lathe operator. At the age of ten he had his right forearm amputated after a bomb accident. The stump measures seventeen centimetres from the tip of the olecranon. One electrode was implanted subcutaneously close to the fascia of the flexor muscles in the stump. The function of the electrode was tested one week after implantation and thereafter at monthly intervals. After five months the electrode was removed for technical control and histological examination.

Case 4-The second below-elbow amputee was a twenty-eight-year-old male painter. At the age of eleven his left forearm was sawn through. The length of the stump is now seventeen centimetres from the tip of the olecranon. One electrode was implanted subcutaneously close to the fascia of the extensor muscles in the stump. The function of the electrode was tested after one week and then regularly every month. After three months the electrode was removed for technical control and histological examination in the same way as the others.

\section{SURGICAL PROCEDURE}

All the implantations and removals were performed under local anaesthesia. The necessary incision is approximately two centimetres in length. The muscle fascia is cleaned and the electrode placed with the gold wires along the fibre axis of the muscle immediately 
superficial to the fascia. The operation takes about fifteen minutes. An electromyographic investigation is performed before the operation in order to ascertain the best position for the electrode.

\section{TEST PROCEDURE}

A measure of the contraction level in the muscle concerned has been obtained by applying loads to the relaxed hand at the metacarpo-phalangeal joints (Fig. 5). The myo-potentials detected in the external receiver were recorded on tape for further analysis. The recording and analysing procedures are indicated in Figure 6. The detected myo-electric potentials are amplified (Disa Electromyograph 13 A 69) and recorded on tape via frequency modulation (Precision Instruments PI-200). The recording is made with a tape velocity of 45 centimetres per second in the frequency range $0-5 \mathrm{kHz}$. As an alternative, analysis is performed with the aid of the "locus-analyser" described by Kaiser and Petersen $(1963,1965)$. They showed that the amplitude spectrum of myo-potentials from the face and from the limbs can be expressed via the activity in the octave bands with centre frequencies 50,200 and $800 \mathrm{~Hz}$ respectively. By using a coordinate system for display of data, the locus of a single spot (the locus point) is sufficient for a rough estimation of the spectrum. The ordinate of a point in Figure 7 indicates by how many dB the $200 \mathrm{~Hz}$

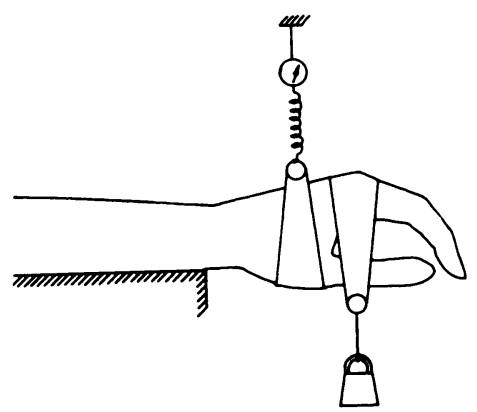

FIG. 5

Schematic figure of the experimental set-up in the contraction level tests. A dynamometer compensates for the weight of the hand. filter response exceeds the $50 \mathrm{~Hz}$ filter response. Similarly, the abscissa expresses the $200 \mathrm{~Hz}$ filter response over the $800 \mathrm{~Hz}$ and the $1,600 \mathrm{~Hz}$ filter responses respectively. The following methods of analysis of recorded potentials have been used (Fig. 6): 1) study via rectification and integration of the total signal; 2) spectral analysis; 3) study via graphic recording.
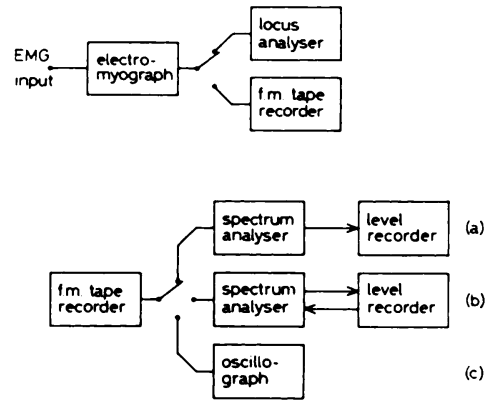

Fig. 6

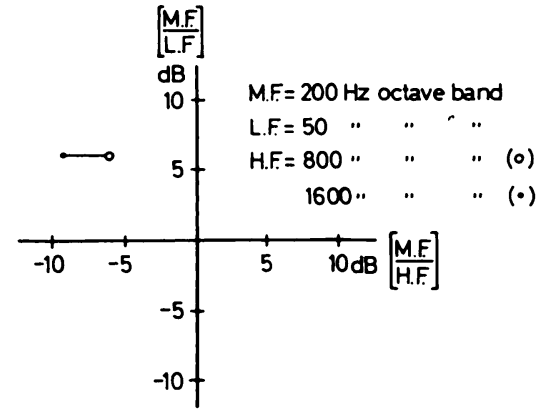

Fig. 7

Figure 6-Block diagram showing the recording and analysis procedure (see text). Method $(a)$ gives a rectified and integrated signal, $(b)$ a spectrum, and $(c)$ a graphic recording of the myo-potentials. Figure 7-Locus diagram. The ordinate expresses the relative content of signal in $200 \mathrm{~Hz}$ and $50 \mathrm{~Hz}$ octave bands respectively. Similarly, the abscissa expresses $(a)$ the relative content of signal in the $200 \mathrm{~Hz}$ and $800 \mathrm{~Hz}$ octave bands (circle), (b) $200 \mathrm{~Hz}$ and 1,600 $\mathrm{Hz}$ octave bands (filled dot). The locus points in the figure pertain to the case of white noise.

Graphic recording of myo-potentials is usually made in real time, thus making heavy demands upon the recorder bandwidth. In the present study analysis has been undertaken by using a tape recording velocity of eight times the playback velocity. All frequencies are thus divided by eight. A conventional ink-jet recorder (Elema Schönander Mingograf 42) capable of writing frequencies up to some hundreds $\mathrm{Hz}$ is adequate for the display. Rectification and integration of the total signal have been performed by the spectrum analyser, also offering frequency analysis facilities (Brüel and Kjaer 2112). This instrument consists of an amplifier,

VOL. $50 \mathrm{~B}$, NO. 4, NOVEMBER 1968 
a bank of octave-band filters and a detector. The filter output terminals can be scanned and the signals fed to an internal detector or to a level recorder (Brüel and Kjaer 2305) which also includes circuits for amplification and detection. Operated in the scanning mode, the analyser gives the frequency spectrum of the signal in the $11-40,000 \mathrm{~Hz}$ band.

\section{RESULTS}

Clinical results--The volunteers have in no way found the surgical procedures unpleasant or painful. Slight aching was experienced in Cases 1 and 2 during the first twenty-four hours after the local anaesthesia had ceased. All subjects returned to full work the day after the surgical intervention and in Case 1 this involved heavy manual labour. The wounds healed by primary intention and without any complications. One of the amputees (Case 3 ) performed heavy duties the day after operation, and although this involved the use of a leather prosthesis which pressed on the electrode, only slight superficial inflammation was produced. After two weeks the wound showed no reaction. The other amputee (Case 4) worked immediately after the operation with slight pain during the first day. This amputee only sporadically used a cosmetic prosthesis and the wound healed without any complications.

The patients have not been inconvenienced by the implanted electrodes. After the wounds had healed no reaction whatever was clinically observed. The skin maintained normal colour and was freely movable over the electrode, which could be felt without pain. Removal of the electrodes was also performed under local anaesthesia, and the post-operative course has been uneventful except for small haematomata in Cases 2 and 3. All the scars have been very thin and cosmetically acceptable.

At the removal operations the subcutaneously situated electrodes were dissected free from an enveloping connective-tissue membrane which occasionally was slightly adherent to the skin but not to the underlying fascia. Macroscopically there were differences between the tissue reactions observed around the electrodes in the four cases. In the case in which the electrode was implanted for the longest time (Case 1, fifteen months) a thin and pale fibrous capsule surrounded both electrode and gold wires. In Case 4 there was a more pronounced reaction with a thicker, more vascular membrane. Macroscopically there had thus been an obvious tissue reaction with the development of a fibrous capsule vascularised to a varying degree. These observations point to differing stages of inflammatory reaction.

The tissues for histological examination consisted of capsular connective tissue with a cystic defect. The specimens were embedded in paraffin and cut in sections $3 \mu$ thick. They were stained by Weigert's haematoxylin-van Gieson technique, and, for iron, by the conventional Prussian blue technique. The microscopic picture was almost the same in all cases. There was a capsule of granulation tissue of varying thickness (Fig. 8). There were layers of histiocytic cells on the surface and some foreign-body giant cells (Fig. 9). In one case there were foreignbody granulomas in the neighbourhood of the capsule. In every case iron pigment was observed in the capsule and in the connective tissue around it and in one case (Fig. 10) was probably too much to be only endogenous. Small amounts of fibres and granules with foreign-body cell reaction were also found (Fig. 11). The granulation tissue showed inflammatory reaction with a slight diffuse infiltration by lymphocytes. No vascular changes were observed.

Microscopical examination has thus shown that a capsule of granulation tissue of varying thickness develops around the implanted electrode and that it shows a slight inflammatory reaction with foreign-body giant cells.

The transmitted myo-potentials-The signals received from the implanted electrodes have been of high quality in all cases except in Case 4 in which distinct signals were never detected. We will return to this below.

As an example, Figure 12 shows signals from a normal subject (Case 1) as received during the first twenty-four hours after the operation and fifteen months after. According to 


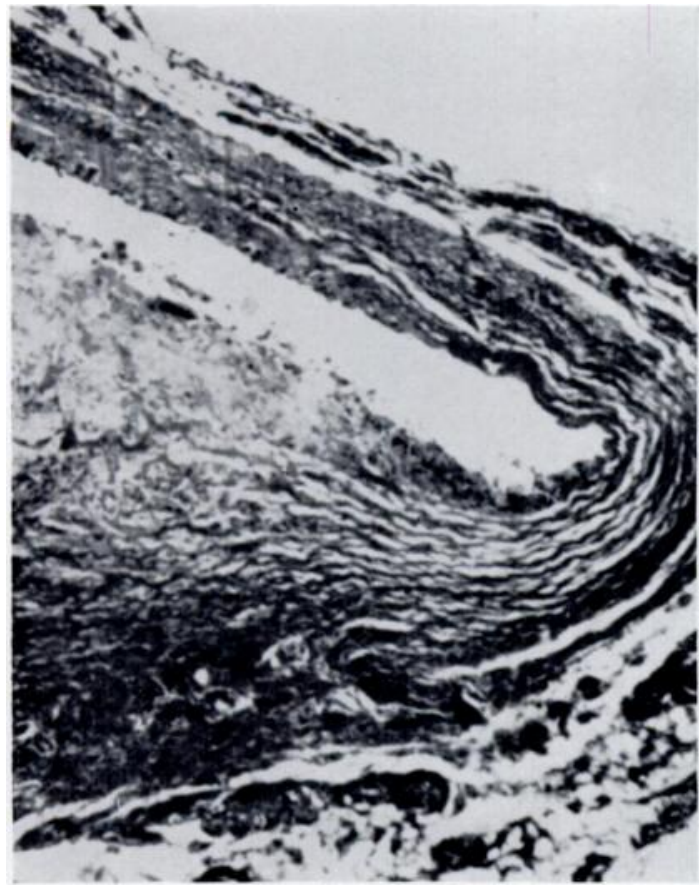

Fig. 8

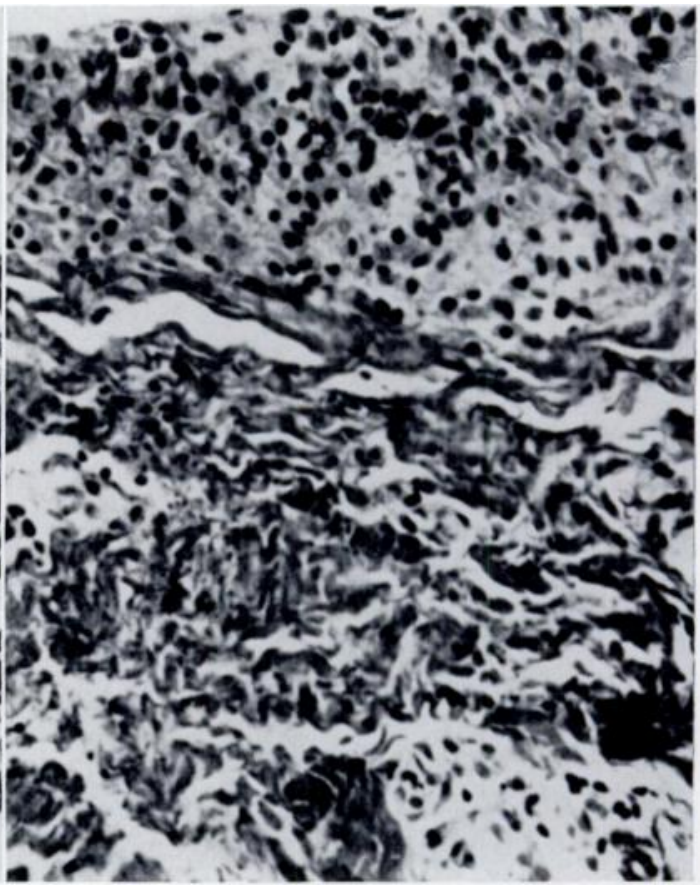

Fig. 9

Figure 8-Capsule, from Case 3, of varying thickness consisting of connective tissue with a slight inflammatory reaction. (Weigert's haematoxylin-van Gieson method $\times 40$.) Figure 9-Histiocytic cells in several layers on the surface of the granulation tissue capsule around the proximal electrode in Case 1. (Weigert's haematoxylinvan Gieson method, $\times 192$.)

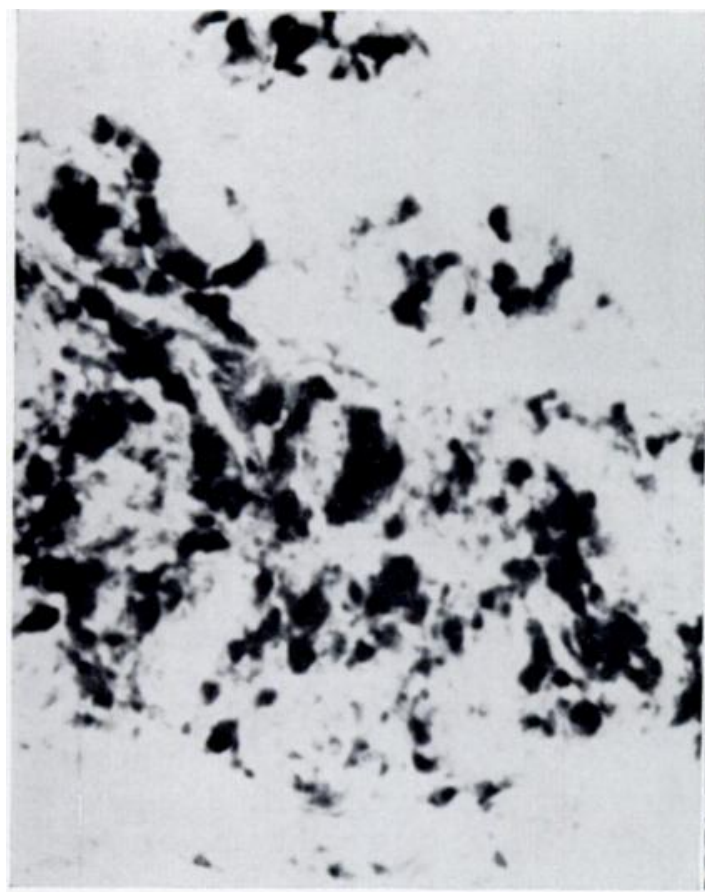

FIG. 10

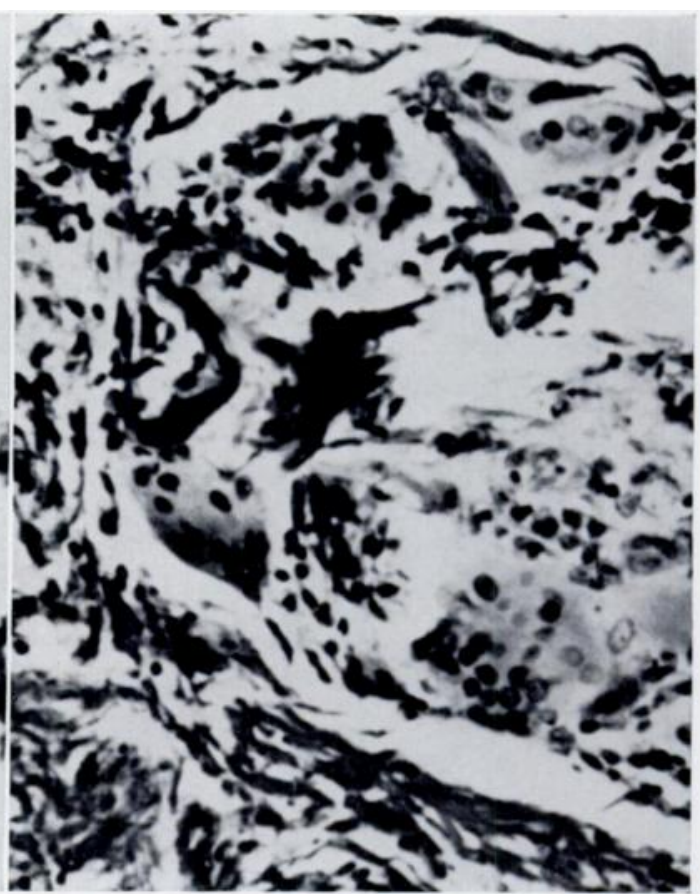

FIG. 11

Figure 10-Granulation tissue capsule with iron pigment. (Prussian blue around the proximal electrode in Case 1, $\times 192$, ) Figure 11-Granuloma with multinucleated foreign-body giant cells and needle-shaped monorefringent material in Case 4. (Weigert's haematoxylin-van Gieson method, $\times 192$.

VOL. SO B, NO. 4, NOVEMBER 1968 
conventional electromyographic evaluation these signals are considered to be normal and of high technical quality. In Figure 13 signals from a below-elbow stump muscle (Case 3) are shown. These signals were detected seven weeks after the implantation.

It has been shown (Bigland and Lippold 1954; Kaiser, Kadefors, Magnusson and Petersén 1968) that the relation between muscle force and the total integrated EMG is almost linear for isometric contractions. This relationship is illustrated in Figure 14, which shows transmitted myo-potentials at different levels of contraction (Case 1, proximal electrode). The signals were recorded six months after the implantation. If they are compared with the signals in Figure 12 it can be concluded that the electrode provides signals which are reproducible in shape.

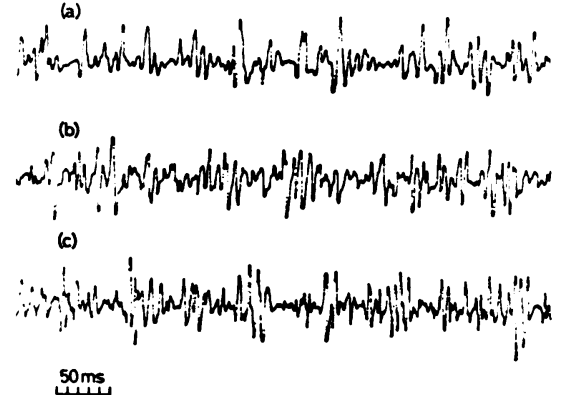

FIG. 12

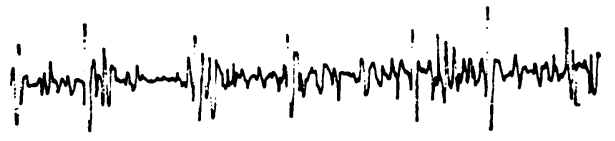

FIG. 13

FIGs. 12 AND 13

Figure 12-Transmitted myo-potentials from the normal forearm of Case 1 in strong contraction: (a) signals from the proximal transmitter; (b) signals from the distal transmitter. The recordings were made during the first day after the implantation. (c) Signals from the distal transmitter fifteen months later. Figure 13-Transmitted myo-potentials from the flexor side of the below-elbow stump of Case 3. The signals were recorded seven weeks after implantation.

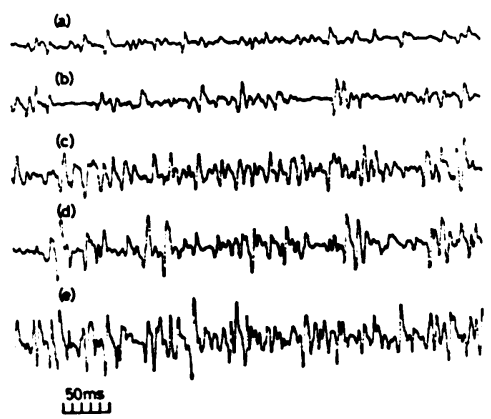

FIG. 14

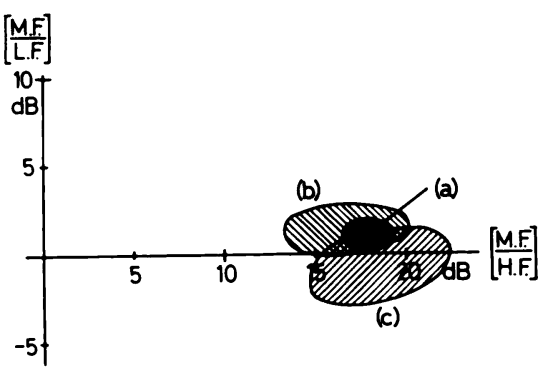

FIG. 15

Figure 14-Transmitted signals from the normal forearm of Case 1 with different loads applied at the hand: (a) $1 \mathrm{kp},(b) 2.5 \mathrm{kp},(c) 5 \mathrm{kp},(d) 10 \mathrm{kp}$, (e) maximum voluntary contraction. Correction has been made for the weight of the hand. The signals were recorded six months after the implantation. Figure 15-Locus diagram (see Fig. 7) of telemetered signals from the forearm of Case 1 in a low level of contraction: $(a)$ the first day after the implantation, $(b)$ four weeks later, $(c)$ four months later.

A quantitative measure of the reproducibility can be obtained by frequency analysis as described above (Fig. 7). There are no significant modifications to be found in the frequency spectrum as a function of time after the implantation (Fig. 15). It should be emphasised that the spectrum is almost independent of the contraction level (Kaiser et al. 1968). In fact, a considerable change in the contraction level causes a minor change of the profile as shown in Figure 16 (Case 2). These findings thus indicate that it is possible to obtain proportional control of powered prostheses with the aid of implanted circuits over long periods of time.

The statement above that the signals detected are of high quality and as good as those obtained by conventional electromyography is supported by Figure 17, which shows simultaneously recorded signals (Case 1) from an implanted electrode and a coaxial electrode 
(diameter 0.65 millimetre, inner connector area 0.07 square millimetre) inserted in the same muscle. In this figure the needle electrode recording seems to have a higher noise level than has the implanted electrode recording. The relevance of this impression is demonstrated in Figure 18, which shows the signal-to-noise ratio of these recordings. The muscle was first loaded by a small weight at the hand ("contraction "). When the weight was removed, the recording continued for about eight seconds (" rest"). Then the tape recorder was switched off (" off "). The difference in dB between the " contraction " and the " rest "levels is a measure of the signal-to-noise ratio pertaining to this particular experiment and offers a possibility to compare electrodes. The figure shows that the implanted circuit provided signals with an improvement in the signal-to-noise ratio of about $3 \mathrm{~dB}$ as compared to the needle electrode

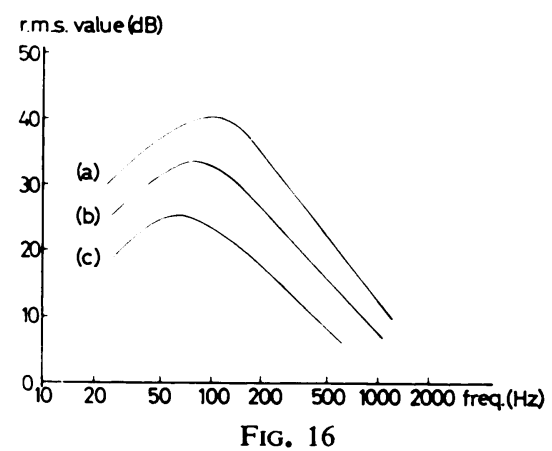
$(c) 2 \cdot 5 \mathrm{kp}$ weight applied at the hand. (For
method see Figure $6(b)$.) The recording was made three months after the implantation.
Figure 17 -Simultaneously recorded signals from: $(a)$ an implanted electrode, $(b)$ a coaxial Figure 17-Simultaneously recorded signals from: $(a)$ an implanted electrode, $(b)$ a coaxial needle electrode inserted into the muscle close to the implant in Case 1. The recording was made ten months after the implantation.
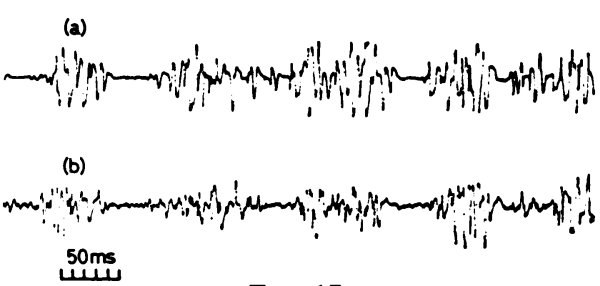

FIG. 17

Figs. 16 AND 17

Figure 16-Amplitude spectra of transmitted signals at different levels of load from the flexor signals at different levels of load from the flexor
side of the forearm of Case $2:(a) 10 \mathrm{kp},(b) 5 \mathrm{kp}$.

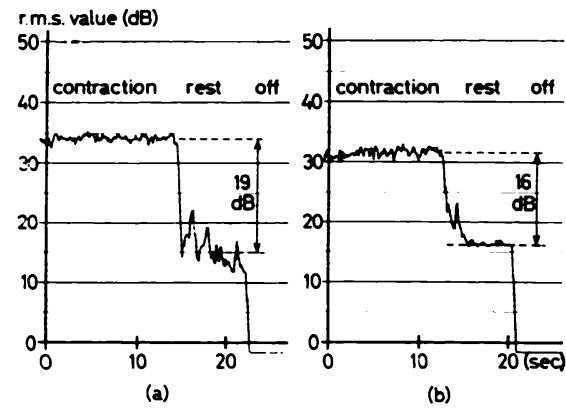

FiG. 18

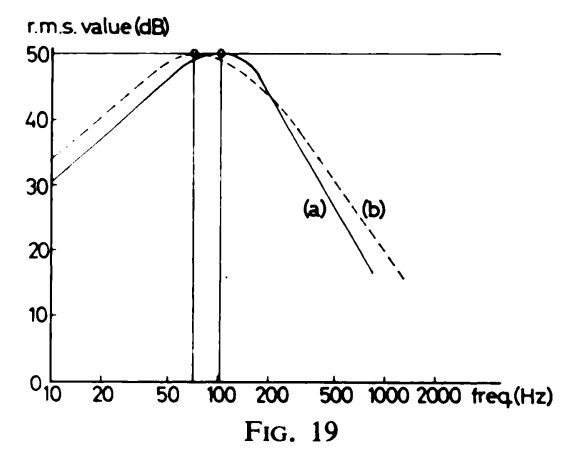

Figure 18-Square-law rectified and average signals from: $(a)$ an implanted electrode, $(b)$ a coaxial needle electrode recorded as in Figure 17. (For method see Figure $6(a)$.) Figure 19-Spectra of signals from: $(a)$ an implanted electrode in Case $1,(b)$ a dry surface electrode taken under equivalent experimental conditions. The curves are made normal to their maxima of $50 \mathrm{~dB}$.

(For method see Figure $6(b)$.) The recordings were made six months after the implantation.

signals. As a matter of fact there is still certain motor-unit activity during the "rest" state not within the needle electrode pick-up area but present in the implanted electrode signal, indicating that the signal-to-noise ratio might be still more favourable.

One of the problems associated with skin electrode signals is their low content of highfrequency power. As discussed by Kaiser et al. (1968) large signal bandwidth is essential for the best proportional control of prostheses. In Figure 19 amplitude spectra for signals from an implanted electrode (Case 1, six months after the operation) and a dry surface electrode (Viennatone) are compared. The implanted electrode curve reaches its maximum at a somewhat higher frequency than does the surface electrode curve. The reason for the steeper fall of the implanted electrode curve at higher frequencies will be discussed below.

VOL. 50 B, NO. 4, NOVEMBER 1968

G 


\section{TECHNICAL ASPECTS ON THE DESIGN OF THE ELECTRODES}

A most essential problem in the design of implantable electronic circuits is how to protect the components from contact with the surrounding tissues and the body fluids. In this investigation no technical failure has been due to insufficient encapsulation of the semiconductors.

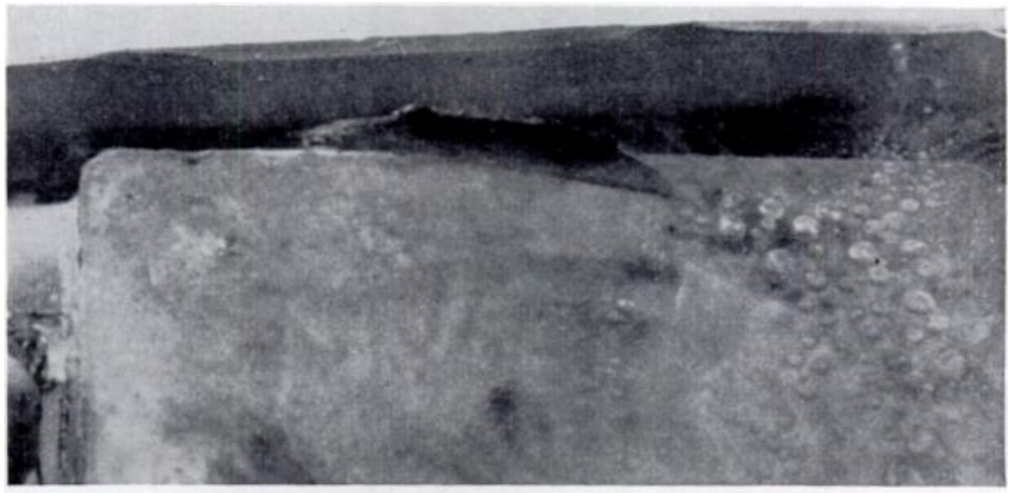

Fig. 20

A crack has occurred between the flat-pack edge and the surface of the plastic layer. (From Case 4.)

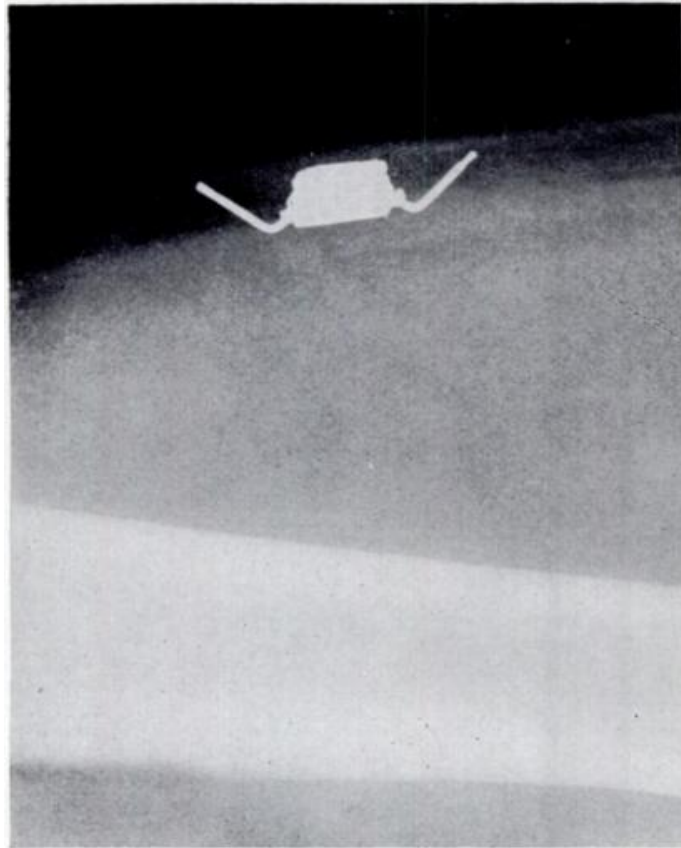

Fig. 21

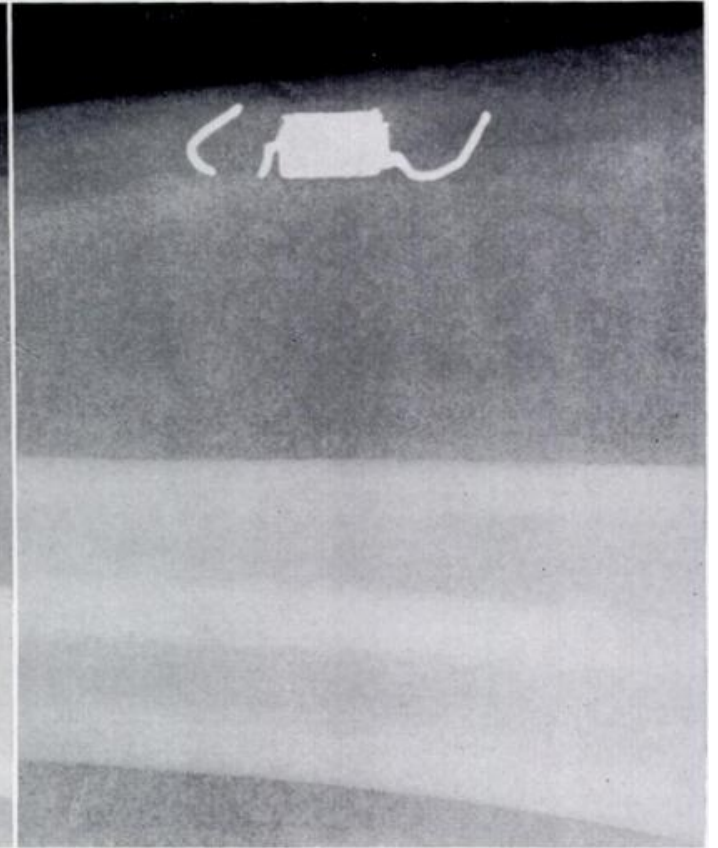

FIG. 22

Figure 21-Radiograph of the distal implanted electrode in Case 1, which was still operating after fifteen months. Figure 22-Radiograph of the implanted electrode on the extensor side of Case 2 after fatigue and breakage of the gold wire at the surface of the plastic layer. The plastic is invisible in the radiograph.

In the present application the plastic layer is not protecting the electronic components from diffusion of water vapour; it serves essentially as a mechanical stabilising material. A general impression has been that the plastic is excellent for the purpose if it is applied in layers of a certain thickness. In three cases out of six, however, minor cracking occurred at the edges 
of the flat-pack, as shown in Figure 20. In no case has this effect caused a failure, but as clear traces of metal and body fluid are found in the cracks, it is a potential site of contact between the tissue and embedded metal material.

Apart from cracking and some discoloration, the plastic has been unaffected by the influence of the body environment. The curing shrinkage has been small and has not caused any wire to break.

Five transmitters out of six have been removed because of failures. One transmitter (Case 1, distal one) was still operating fifteen months after implantation. A radiograph of this electrode is shown in Figure 21 ; note the upward bending of the gold wires.

In three cases failure has been caused by fatigue fractures in the gold wires at the surface of the plastic layer (Case 2, both electrodes, and Case 3). Figure 22 shows a radiograph of one of these failures (Case 2, extensor side). Radiography was undertaken when a regular test showed unsatisfactory operation of the electrodes.

In Case 1 the proximal electrode was removed for examination of the tissue reactions after a test in which no signals were received. As the transmitter operated perfectly after its removal, this failure has been attributed to inadequate operation of the external part of the system.

In Case 4 distinct signals were never detected. Examination of the electrode after removal showed a breakdown in the electronic circuit, the reason for which probably lies with the hybrid technology used in the micro-circuit design.

The external energy supply for the implanted transmitters has been reliable. As the magnetic field supplied by the energy transmitter decays with increasing distance, the efficiency of the energy transmission to the implanted electrode has been rather critical, regarding the relative positioning of the implanted and the external circuits.

The transmission of myo-potentials from the implanted electrode has been satisfactory. The signals can be detected at a distance of several metres from the electrode. The influence of the main supply on the signal has been negligible in the buried system.

\section{DISCUSSION}

The results will be discussed in accordance with the list of demands to be met by implantable electrode as formulated in the introduction.

One of the primary problems in connection with implantations is to find a protecting material of low toxicity, causing a minimum of foreign-body reaction. The plastic material used in this investigation has been embedded in a connective-tissue capsule of varying thickness. Histologically there has been slight inflammatory reaction in all cases, especially in Case 4, in which there was also a marked foreign-body reaction. In this case a crack in the plastic material had occurred, permitting free diffusion of metal ions to the surrounding tissue (Figs. 11,12 and 21). The needle-shaped monorefringent material found (Fig. 12) might be plastic.

The complete absence of clinical symptoms in the volunteers together with the histologically slight inflammatory reactions shows that the toxicity of the material has been low.

The results indicate that implantation in human beings with the plastic chosen seems to be tolerated over a period of a year. However, certain reactions have occurred which indicate that further tests with other materials should be carried out. A possible solution is to use the plastic in combination with another material, for example, silicon rubber (Roberts 1967). Unfortunately the electrical environment of the embedded circuits is rapidly influenced by diffusion of water vapour through the rubber layer (Balin et al. 1964). Also, this material has little mechanical stiffness and this is a drawback in the present application. Regarding the carcinogenic effect of the plastic material, no definite conclusions can be drawn. However, no malign signs have been found in the histological examinations. From the technical point of view, no failures have occurred from inadequate mechanical or chemical protection in this investigation.

VOL. 50 B, NO. 4, NOVEMBER 1968 
The operative procedure has in no way been painful or unpleasant to the patients and has been easily performed under local anaesthesia. The scars left have in all cases been thin and cosmetically acceptable.

The signals detected from the implanted electrodes have been reliable and reproducible over long periods of time, both in shape and in frequency spectrum. It seems thus to be clear that no progressive shunting effect occurs as a result of encapsulation of transmitter by the tissue.

The most important source of failure of the implantable electrodes has been the fatigue breakdown of the gold wires. These failures can readily be overcome by using very thin and flexible wires for the tissue connection (Caldwell 1967). Such modifications are under development. The myo-potentials have been of high quality, especially regarding the signal-tonoise ratio. This fact can be attributed partly to the low-noise amplification taking place in the modulation procedure, which makes it superior, especially at low levels of contraction, to the conventional electrode technique. At higher levels of contraction the frequency spectrum of myo-potentials detected with implantation technique differs little from the corresponding surface EMG spectrum (Fig. 19). It should be emphasised that the ability to detect highfrequency components in the signals is partly limited by inadequate configuration of the gold wires. A limiting effect occurring in the implantable electrode system is the restricted bandwidth of the receiver. In our experiments this effect limits the high-frequency detection as was shown in Figure 19. By increasing the receiver bandwidth this drawback can be eliminated.

From the technical point of view, the miniaturisation achieved is adequate. Further progress in miniaturisation is limited so long as the present method for external energising is employed. On the other hand, it is possible to obtain increased reliability with the aid of refined integrated circuit technology.

The method of energising the implanted electrode is considered to be adequate for the purpose. The battery cells required are already used in electric prosthesis systems.

\section{SUMMARY}

1. Experience with a refined type of implantable electrode for the myo-electric control of externally powered prostheses is reported.

2. The electrodes are externally energised by electromagnetic induction and therefore do not contain any battery cells. The myo-potentials are transmitted in frequency-modulated form and detected by a receiver placed on the skin. The implantable electrode, measuring $5 \times 11 \times 4$ millimetres, is encapsulated in epoxy resin.

3. Six electrodes have been implanted in the forearms of two normal subjects and two below-elbow amputees. The time of implantation ranged from three to fifteen months. Macroscopically, a fibrous capsule developed around the electrodes. Histological examination showed a capsule of granulation tissue of varying thickness with slight inflammatory reaction and foreign-body giant cells.

4. In all cases except one the signals received have been of high quality as ascertained by conventional electromyography and frequency analysis. There has been no significant deterioration in signal quality during the follow-up periods.

5. The major source of failure was fatigue fracture of the gold wires making contact with the body tissues. In one case, however, the electrode was still functioning normally at the time of removal fifteen months after insertion.

6. The patients have not been inconvenienced either by the operative procedures or by the presence of the electrodes in the tissues.

We want to express our gratitude to Dr G. Hansson of the Institution of Pathology, University of Göteborg, for the microscopical examination of tissue membranes surrounding the implants.

This investigation has been supported by grants from The Swedish Council of Applied Research, The Medical Research Council and The Insurance Company, Folksam. 


\section{REFERENCES}

Balin, H., Busser, J. H., Hatke, F., Fromm, E., Wan, L. S., and Israel, S. L. (1964): Radio Telemetry System for the Study of Ovarian Physiology. Obstetrics and Gynecology, 24, 198.

Basmajian, J. V. (1967): Muscles Alive: Their Functions Revealed by Electromyography. Second edition. Baltimore: Williams and Wilkins Co.

Battye, C. K., Nightingale, A., and Whillis, J. (1955): The Use of Myo-electric Currents in the Operation of Prostheses. Journal of Bone and Joint Surgery, 37-B, 506.

Bigland, B., and LipPOLD, O. C. J. (1954): The Relation between Force, Velocity and Integrated Activity in Human Muscles. Journal of Physiology, 123, 214.

Caldwell, C. (1967): A New Transcutaneous Electrode. In Some Topics on Myo-electric Control of Orthoticprosthetic Systems. Report No. EDC 4-67-17, p. 124. L. Vodovnik, ed., Engineering Design Centre, Case Western Reserve University, Cleveland, Ohio.

Hirsch, C., Kadefors, R., Kaiser, E., and Petersén, I. (1967): An Implantable Microcircuit for Long Term Telemetry of Myo-potentials. Digest of the 7th International Conference on Medical and Biological Engineering, Stockholm, p. 365.

Hirsch, C., Kaiser, E., and Petersén, I. (1964): Bioelectrical Control in a Servo-system. Acta Orthopaedica Scandinavica, 35, 1.

Hirsch, C., KaISer, E., and Petersén, I. (1966): Telemetry of Myo-potentials. Acta Orthopaedica Scandinavica. $37,156$.

Kadefors, R., Herberts, P., Hirsch, C., Kaiser, E., and Petersén, I. (1968): Electrodes for Myo-electric Control of Prostheses. P. 43, In Medicinsk Teknik 1, Swedish Council for Applied Research, Stockholm.

Kadefors, R., Kaiser, E., and Petersén, I. (1968): Methods for Studying the Modifications in Frequency Spectrum of Myo-potentials at Slow and Fast Muscle Contractions. Electroencephalography and Clinical Neurophysiology, 24, 89.

Kaiser, E., Kadefors, R., Magnusson, R., and Petersén, I. (1968): Myoelectric Signals for Prosthesis Control. P. 14. Myo-electric Control of Prostheses, Swedish Council for Applied Research, Stockholm.

Kaiser, E., and Petersén, I. (1963): Frequency Analysis of Muscle Action Potentials during Tetanic Contraction. Electromyography, 3, 5.

Kaiser, E., and Petersén, I. (1965): Muscle Action Potentials Studied by Frequency Analysis and Duration Measurement. Acta Neurologica Scandinavica, 41, Suppl. 13, 213.

Ko, W. H. (1964): Progress in Telemetering Muscle Potentials. In Biomedical Sciences Instrumentation. W. E. Murray and P. F. Salisbury, eds. New York: Plenum Press.

Kobrinski, A. E., Bolkhovitin, S. V., Voskoboinikova, L. M., Ioffe, D. M., Polyan, E. P., Slavutski, Ya. L., SYsin, A. Ya., and YAKobson, Ya. S. (1961): Proceedings of the First International Congress of the International Federation of Automatic Control (I.F.A.C.). Moscow, 1960. pp. 1119-1122. J. F. Coales, Editor. London: Butterworths.

RoberTs, A. C. (1967): Silicones and their Application as Implant Materials. Bio-Medical Engineering, 2, 156.

Scotr, R. N. (1965): A Method of Inserting Wire Electrodes for Electromyography. IEEE Transactions on Bio-Medical Engineering, BME-12, 46. 